\title{
THREE PARIETAL CIRCUITS FOR NUMBER PROCESSING
}

\author{
Stanislas Dehaene, Manuela Piazza, Philippe Pinel, and Laurent Cohen \\ INSERM-CEA, Service Hospitalier Frédéric Joliot, Orsay, France
}

\begin{abstract}
Did evolution endow the human brain with a predisposition to represent and acquire knowledge about numbers? Although the parietal lobe has been suggested as a potential substrate for a domain-specific representation of quantities, it is also engaged in verbal, spatial, and attentional functions that may contribute to calculation. To clarify the organisation of number-related processes in the parietal lobe, we examine the three-dimensional intersection of $\mathrm{fMRI}$ activations during various numerical tasks, and also review the corresponding neuropsychological evidence. On this basis, we propose a tentative tripartite organisation. The horizontal segment of the intraparietal sulcus (HIPS) appears as a plausible candidate for domain specificity: It is systematically activated whenever numbers are manipulated, independently of number notation, and with increasing activation as the task puts greater emphasis on quantity processing. Depending on task demands, we speculate that this core quantity system, analogous to an internal "number line," can be supplemented by two other circuits. A left angular gyrus area, in connection with other left-hemispheric perisylvian areas, supports the manipulation of numbers in verbal form. Finally, a bilateral posterior superior parietal system supports attentional orientation on the mental number line, just like on any other spatial dimension.
\end{abstract}

\section{INTRODUCTION}

Did evolution endow the human brain with a predisposition to represent dedicated domains of knowledge? We have previously argued that the number domain provides a good candidate for such a biologically determined semantic domain (Dehaene, 1997; Dehaene, Dehaene-Lambertz, \& Cohen, 1998a). Three criteria for domain specificity suggest that number and arithmetic are more than cultural inventions, and may have their ultimate roots in brain evolution. First, a capacity to attend to numerosity, and to manipulate it internally in elementary computations, is present in animals even in the absence of training (Hauser, Carey, \& Hauser, 2000). Second, a similar capacity for elementary number processing is found early on in human development, prior to schooling or even to the development of language skills (Spelke \& Dehaene, 1999; Xu \& Spelke, 2000). This suggests that numerical development follows a distinct developmental trajectory based on mechanisms with a long prior evolutionary history.

Third, it has been suggested that number processing rests on a distinct neural circuitry, which can be reproducibly identified in different subjects with various neuroimaging, neuropsychological, and brain stimulation methods (Dehaene et al., 1998a). The present paper focuses on this last issue, taking into account the considerable progress that has recently been made in neuroimaging methods. The involvement of parietal cortex in number processing was initially discovered on the basis of lesion data (Gerstmann, 1940; Hécaen, Angelergues, \&

Requests for reprints should be addressed to Stanislas Dehaene, INSERM Unit 562, Service Hospitalier Frédéric Joliot, CEA/ DRM/DSV, 4 place du Général Leclerc, 91401 Orsay cedex, France (Email: dehaene@shfj.cea.fr). 
Houillier, 1961; Henschen, 1919). Subsequently, a systematic activation of the parietal lobes during calculation, together with precentral and prefrontal cortices, was discovered (Roland \& Friberg, 1985) and extensively replicated using positron emission tomography (PET) (Dehaene et al., 1996; Pesenti, Thioux, Seron, \& De Volder, 2000; Zago, Pesenti, Mellet, Crivello, Mazoyer, \& Tzourio-Mazoyer, 2001) and later fMRI (Burbaud, Camus, Guehl, Bioulac, Caille, \& Allard, 1999; Rueckert et al., 1996). On this basis, some of us proposed that the parietal lobe contributes to the representation of numerical quantity on a mental "number line" (Dehaene \& Cohen, 1995). Unfortunately, due to poor spatial resolution and limits on experimental designs, those studies did not permit a finer exploration of the regions involved in different kinds of numerical tasks. This has become critical, however, because recent behavioural studies have made it clear that mental arithmetic relies on a highly composite set of processes, many of which are probably not specific to the number domain. For instance, studies of language interference in normal subjects suggest that language-based processes play an important role in exact but not approximate calculation (Spelke \& Tsivkin, 2001). Likewise, concurrent performance of a spatial task interferes with subtraction, but not multiplication, while concurrent performance of a language task interferes with multiplication, but not subtraction (Lee \& Kang, 2002). Such behavioural dissociations suggest that the neural bases of calculation must be heterogeneous.

The triple-code model of number processing predicts that, depending on the task, three distinct systems of representation may be recruited: a quantity system (a nonverbal semantic representation of the size and distance relations between numbers, which may be category specific), a verbal system (where numerals are represented lexically, phonologically, and syntactically, much like any other type of word), and a visual system (in which numbers can be encoded as strings of Arabic numerals) (Dehaene, 1992; Dehaene \& Cohen, 1995). We initially proposed that the parietal activations during number processing reflected solely the contribution of the quantity system. However, it is now clear that this hypothesis requires further elaboration. First, the left perisylvian language network clearly extends into the inferior parietal lobe. Second, the posterior superior parietal lobes are strongly engaged in visual attention processes that may contribute to the visual processing of numbers. It is thus crucial to distinguish, within the observed parietal lobe activations during number processing, which activation sites, if any, are associated with a semantic representation of numerical quantity and which correspond to nonspecific verbal or visual/ attentional systems.

Fortunately, functional magnetic resonance imaging (fMRI) has recently allowed much finergrained studies of the neuroanatomy of number processing, using paradigms adapted from cognitive psychology. The present review focuses entirely on the parietal lobe activations identified by those recent neuroimaging studies. We use three-dimensional visualisation software to investigate how the parietal activations reported by various studies relate to one another in cortical space. On this basis, we propose that three circuits coexist in the parietal lobe and capture most of the observed differences between arithmetic tasks: a bilateral intraparietal system associated with a core quantity system, a region of the left angular gyrus associated with verbal processing of numbers, and a posterior superior parietal system of spatial and nonspatial attention.

It should be emphasised that our description provides only a tentative model. Although it is based on a synthesis of the existing literature, this model remains speculative and will require further validation by direct experimentation. For each postulated circuit, we first examine the relevant neuroimaging literature, and then consider how those brain-imaging results impinge on our understanding of neuropsychological impairments of number processing. Our account predicts that depending on lesion localisation, three different categories of numerical impairments should be observed: genuine semantic impairments of the numerical domain following intraparietal lesions; impairments of verbal fact retrieval following lesions to the left perisylvian cortices, including the left angular gyrus; and impairments of spatial 
attention on the number line following lesions to the dorsal parietal attention system.

\section{THE BILATERAL HORIZONTAL SEGMENT OF THE INTRAPARIETAL SULCUS AND QUANTITY PROCESSING}

\section{Neuroimaging evidence}

The horizontal segment of the intraparietal sulcus (hereafter HIPS) is a major site of activation in neuroimaging studies of number processing. As shown in Figure 1a, this region lies at the intersection of the activations observed in many different number processing tasks (see Table 1). What seems to be common to those tasks is the requirement to access a semantic representation of the quantity that the numbers represent. We propose that a nonverbal representation of numerical quantity, perhaps analogous to a spatial map or "number line," is present in the HIPS of both hemispheres. This representation would underlie our intuition of what a given numerical size means, and of the proximity relations between numbers. In support of this view, several features of its responsiveness to experimental conditions are worth noting.

Mental arithmetic. The HIPS seems to be active whenever an arithmetic operation calls upon a quantitative representation of numbers. For example, it is more active when subjects calculate than when they merely have to read numerical symbols (Burbaud et al., 1999; Chochon, Cohen, Van de Moortele, \& Dehaene, 1999; Pesenti et al., 2000), suggesting that it plays a role in the semantic manipulation of numbers. Its activation increases, at least in the right hemisphere, when subjects have to compute two addition or subtraction operations instead of one (Menon, Rivera, White, Glover, \& Reiss, 2000). Furthermore, even within calculation, the HIPS is more active when subjects estimate the approximate result of an addition problem than when they compute its exact solution (Dehaene, Spelke, Stanescu, Pinel, \& Tsivkin, 1999). Finally, it shows greater activation for subtraction than for multiplication (Chochon et al., 1999; Lee, 2000). Multiplication tables and small exact addition facts can be stored in rote verbal memory, and hence place minimal requirements on quantity manipulation. Contrariwise, although some subtraction problems may be stored in verbal memory, many are not learned by rote and therefore require genuine quantity manipulations. In another study, relative to five different visuospatial and phonological non-numerical tasks, subtraction was the only task that led to increased activation of the HIPS (Simon, Cohen, Mangin, Bihan, \& Dehaene, 2002).

Number comparison. The HIPS is also active whenever a comparative operation that needs access to a numerical scale is called for. For instance, it is more active when comparing the magnitudes of two numbers than when simply reading them (Chochon et al., 1999). The systematic contribution of this region to number comparison processes is replicated in many paradigms using tomographic imaging (Le Clec'H et al., 2000; Pesenti et al., 2000; Pinel, Dehaene, Riviere, \& LeBihan, 2001; Thioux, Pesenti, Costes, De Volder, \& Seron, 2002) as well as scalp recordings of event-related potentials (Dehaene, 1996). Parietal activation in number comparison is often larger in the right than in the left hemisphere (Chochon et al., 1999; Dehaene, 1996; Pinel et al., 2001). This may point to a possible right-hemispheric advantage in comparison and in other tasks requiring an abstraction of numerical relations (Langdon \& Warrington, 1997; Rosselli \& Ardila, 1989). However, in comparison, the parietal activation, although it may be asymmetric, is always present in both hemispheres, compatible with the observation that numerical comparison is accessible to both hemispheres in split-brain patients (Cohen \& Dehaene, 1996; Seymour, Reuter-Lorenz, \& Gazzaniga, 1994).

Specificity for the number domain. Several studies have reported greater HIPS activation when processing numbers than when processing other categories of objects on non-numerical scales (such as comparing the ferocity of animals, the relative positions of body parts, or the orientation of two 
A.
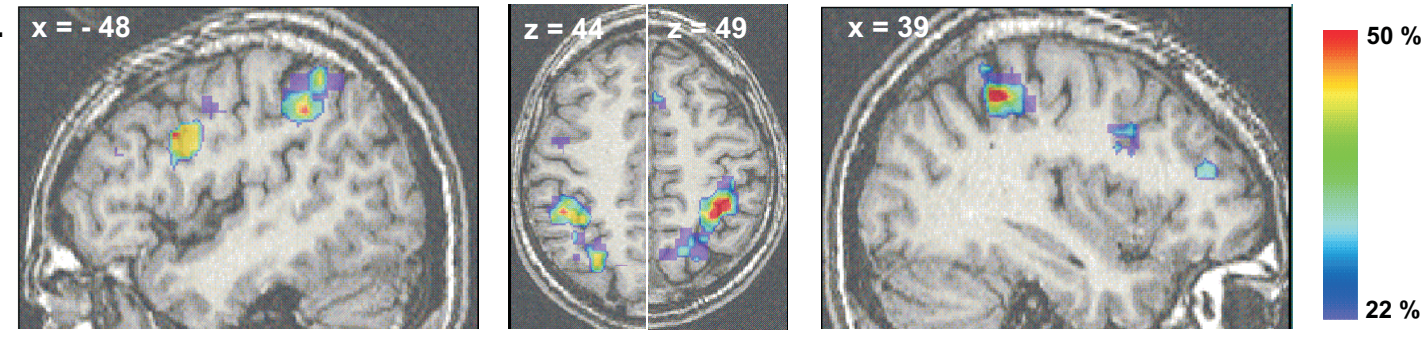

B
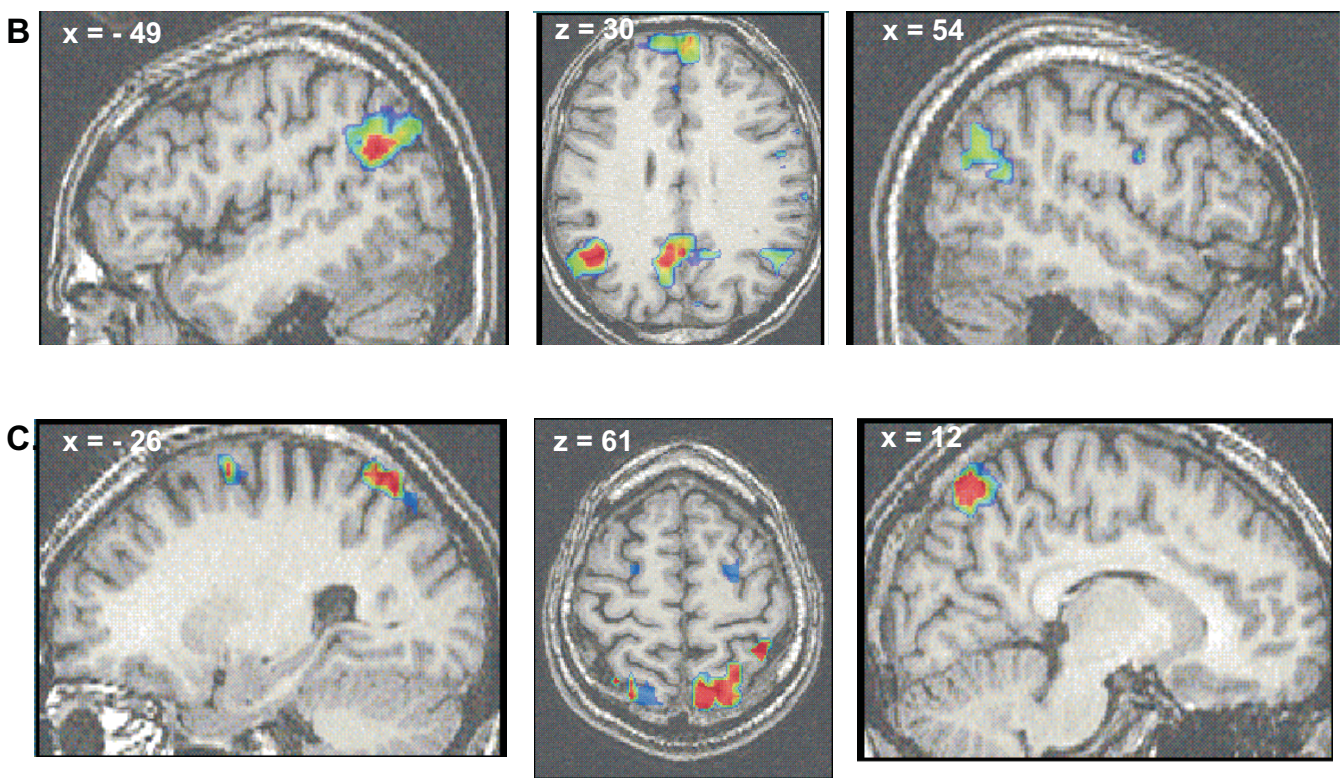

Figure 1. Regions of overlapping activity for three groups of studies, superimposed on axial and sagittal slices of a normalised single-subject anatomical image. The overlap was calculated by averaging binarised contrast images indicating which voxels were significant for a given contrast (studies and contrasts are listed in Table 1). The colour scale indicates the percentage of studies showing activation in a given voxel. The same colour scale (from $22 \%$ to $50 \%$ of overlap) is applied to all images. Although no single voxel was shared by $100 \%$ of studies in a group, probably due to variability across groups of subjects, laboratories, and imaging methods, Table 1 revealed a high consistency of activations. (A) The horizontal segment of the intraparietal sulcus (HIPS) was activated bilaterally in a variety of contrasts sharing a component of numerical quantity manipulation. The barycentre of the region of maximum overlap (>50\%) was at Talairach Coordinates (TC) 41, -42, 49 in the left hemisphere, and -48, -41, 43 in the right hemisphere. Activation overlap is also visible in the precentral gyrus. (B) The angular gyrus $(A G)$ was activated with a strong left lateralisation (TC-48, -59, 30) in 5 studies of arithmetic tasks with a strong verbal component. Posterior cingulate as well as superior frontal regions also show some degrees of overlap. (C) The posterior superior parietal lobule (PSPL) was activated bilaterally in a few numerical tasks (left and right barycentres at TC-26, -69, 61 and 12, -69, 61; and see Table 1). To emphasise the nonspecificity of this region, the image shows the intersection of the overlap between four numerical tasks with an image of posterior parietal activity during a non-numerical visual attention shift task (Simon et al., 2002). 
Table 1. Studies and contrasts used to isolate the three parietal regions in Figures 1 and $2^{a}$

\begin{tabular}{|c|c|c|c|c|c|c|c|}
\hline \multirow[b]{3}{*}{ Reference } & \multirow[b]{3}{*}{ Contrast } & \multicolumn{6}{|c|}{ Coordinates of maxima } \\
\hline & & \multicolumn{3}{|c|}{ Left } & \multicolumn{3}{|c|}{ Right } \\
\hline & & $x$ & $y$ & $z$ & $x$ & $y$ & $z$ \\
\hline & Horizontal segment of intraparietal sulcus (HIPS) & & & & & & \\
\hline Chochon et al. (1999) & Comparison of one-digit numbers vs. letter naming & -45 & -42 & 39 & 39 & -42 & 42 \\
\hline Chochon et al. (1999) & Subtraction of one-digit numbers from 11 vs. comparison & -42 & -48 & 48 & 39 & -42 & 42 \\
\hline Dehaene et al. (1999) & Approximate vs. exact addition of one-digit numbers & -56 & -44 & 52 & 44 & -36 & 52 \\
\hline Lee $(2000)$ & Subtraction vs. multiplication of one-digit numbers & -31 & -52 & 49 & 28 & -54 & 52 \\
\hline Naccache and Dehaene (2001) & Subliminal quantity priming across notations & -44 & -56 & 56 & 36 & -44 & 44 \\
\hline Piazza et al. $\left(2002^{b}\right)$ & Numerosity estimation vs. physical matching & \multicolumn{2}{|c|}{ n.s. } & & 44 & -56 & 54 \\
\hline Pinel et al. (2001) & Distance effect in comparison of two-digit numbers & -40 & -44 & 36 & 44 & -56 & 48 \\
\hline Simon et al. (2002) & Subtraction of one-digit numbers from 11 vs. letter naming & -48 & -44 & 52 & 52 & -44 & 52 \\
\hline Stanescu-Cosson et al. ( 2000 ) & Size effect in exact addition of one-digit numbers & -44 & -52 & 48 & \multicolumn{3}{|c|}{ n.s. } \\
\hline Mean & & -44 & -48 & 47 & 41 & -47 & 48 \\
\hline$S D$ & & 7 & 5 & 6 & 7 & 7 & 5 \\
\hline \multicolumn{8}{|c|}{ Angular gyrus $(A G)$} \\
\hline Chochon et al. (1999) & Multiplication vs. comparison of one-digit numbers & -30 & -69 & 39 & \multicolumn{3}{|c|}{ n.s. } \\
\hline Dehaene et al. (1999) & Exact vs. approximate addition of one-digit numbers & -44 & -72 & 36 & & -76 & 20 \\
\hline Lee $(2000)$ & Multiplication vs. subtraction of one-digit numbers & -49 & -54 & 31 & \multicolumn{3}{|c|}{ n.s. } \\
\hline Simon et al. (2002) & Intersection of subtraction and phoneme detection tasks & -31 & -70 & 43 & \multicolumn{3}{|c|}{ n.s. } \\
\hline Stanescu-Cosson et al. (2000) & Inverse size effect in exact addition of one-digit numbers & -52 & -68 & 32 & \multicolumn{3}{|c|}{ n.s. } \\
\hline Mean & & -41 & -66 & 36 & & & \\
\hline$S D$ & & 9 & 6 & 4 & & & \\
\hline & Posterior superior parietal lobule (PSPL) & & & & & & \\
\hline Dehaene et al. (1999) & Approximate vs. exact addition of one-digit numbers & -32 & -68 & 56 & 20 & -60 & 60 \\
\hline Lee $(2000)$ & Subtraction vs. multiplication of one-digit numbers & -29 & -64 & 69 & 21 & -61 & 65 \\
\hline Naccache and Dehaene (2001) & Subliminal quantity priming across notations & \multicolumn{2}{|c|}{ n.s. } & & 12 & -60 & 48 \\
\hline Pinel et al. (2001) & Distance effect in comparison of two-digit numbers & -4 & -72 & 44 & 8 & -72 & 52 \\
\hline Mean & & -22 & -68 & 56 & 15 & -63 & 56 \\
\hline$S D$ & & 15 & 4 & 12 & 6 & 6 & 8 \\
\hline
\end{tabular}

visually presented characters: Le Clec'H et al., 2000; Pesenti et al., 2000; Thioux et al., 2002). Event-related potentials have also revealed greater parietal activation for numbers than for other categories of words such as action verbs, names of animals, or names of famous persons (Dehaene, 1995). In this study, the first point in time in which category-specific semantic effects emerge during visual word processing was found to be $250-280 \mathrm{~ms}$ following stimulus onset.

One study directly tested the specificity of the HIPS for the numerical domain in multiple tasks
(Thioux et al., 2002). Subjects were presented with number words and names of animals matched for length. The HIPS showed greater activation, bilaterally, to numbers than to animal names. This was true whether subjects were engaged in a comparison task (larger or smaller than 5; more or less ferocious than a dog), a categorisation task (odd or even; mammal or bird), or even a visual judgement of character shape. Thus, the HIPS shows category specificity independently of task context. Further research will be needed, however, to decide whether it is strictly specific for numbers or whether it 
extends to other categories that have a strong spatial or serial component (e.g., the alphabet, days, months, spatial prepositions, etc.).

Parametric modulation. Parametric studies have revealed that the activation of the HIPS is modulated by semantic parameters such as the absolute magnitude of the numbers and their value relative to a reference point. Thus, intraparietal activity is larger and lasts longer during operations with large numbers than with small numbers (Kiefer \& Dehaene, 1997; Stanescu-Cosson, Pinel, Van de Moontele, Le Bihan, Cohen, \& Delaene, 2000). It is also modulated by the numerical distance separating the numbers in a comparison task (Dehaene, 1996; Pinel et al., 2001). On the other hand, the activation of the HIPS is independent of the particular modality of input used to convey the numbers. Arabic numerals, spelled-out number words, and even nonsymbolic stimuli like sets of dots or tones can activate this region if subjects attend to the corresponding number (Le Clec'H et al., 2000; Piazza, Mechelli, Butterworth, \& Price, 2002a; Piazza, Mechelli, Price, \& Butterworth, 2002b; Pinel et al., 2001). In one study, subjects attended either to the numerosity or to the physical characteristics (colour, pitch) of series of auditory and visual events. The right HIPS was active whenever the subjects attended to number, regardless of the modality of the stimuli (Piazza et al., 2002b). In another study, the activation of the bilateral HIPS was found to correlate directly with the numerical distance between two numbers in a comparison task, and this effect was observed whether the numbers were presented as words or as digits (Pinel et al., 2001). Those parametric studies are all consistent with the hypothesis that the HIPS codes the abstract quantity meaning of numbers rather the numerical symbols themselves.

Unconscious quantity processing. Quantity processing and HIPS activation can be demonstrated even when the subject is not aware of having seen a number symbol (Dehaene et al., 1998b; Naccache \& Dehaene, 2001). In this experiment, subjects were asked to compare target numbers to a fixed reference of 5. Unbeknownst to them, just prior to the target, another number, the prime, was briefly present in a subliminal manner. FMRI revealed that the left and right intraparietal regions were sensitive to the unconscious repetition of the same number. When the prime and target corresponded to the same quantity (possibly in two different notations, such as ONE and 1), less parietal activation was observed than when the prime and target corresponded to two distinct quantities (e.g., FOUR and $1)$. This result suggests that this region comprises distinct neural assemblies for different numerical quantities, so that more activation can be observed when two such neural assemblies are activated than when only one is. It also indicates that this region can contribute to number processing in a subliminal fashion.

Taken together, these data suggest that the HIPS is essential for the semantic representation of numbers as quantities. This representation may provide a foundation for our "numerical intuition," our immediate and often unconscious understanding of where a given quantity falls with respect to others, and whether or not it is appropriate to a given context (Dehaene, 1992, 1997; Dehaene \& Marques, 2002).

\section{Neuropsychological evidence}

Neuropsychological observations confirm the existence of a distinct semantic system for numerical quantities and its relation to the vicinity of the intraparietal sulcus. Several single-case studies indicate that numbers doubly dissociate from other categories of words at the semantic level. On the one hand, spared calculation and number comprehension abilities have been described in patients with grossly deteriorated semantic processing (Thioux, Pillon, Samson, De Partz, Noel, \& Seron, 1998) or semantic dementia (Butterworth, Cappelletti, \& Kopelman, 2001; Cappelletti, Butterworth, \& Kopelman, 2001). In both cases, the lesions broadly affected the left temporo-frontal cortices while sparing the intraparietal regions. On the other hand, Cipolotti, Butterworth, and Denes (1991) reported a striking case of a patient with a small left parietal lesion and an almost complete deficit in all spheres of number processing, sparing 
only the numbers 1 through 4 , in the context of otherwise largely preserved language and semantic functions. Although such a severe and isolated degradation of the number system has never been replicated, other cases confirm that the understanding of numbers and their relations can be specifically impaired in the context of preserved language and semantics (e.g., Dehaene \& Cohen, 1997; Delazer \& Benke, 1997).

In many cases, the deficit can be extremely incapacitating. Patients may fail to compute operations as simple as $2+2,3-1$, or $3 \times 9$. Several characteristics indicate that the deficit arises at an abstract, notation-independent level of processing. First, patients may remain fully able to comprehend and to produce numbers in all formats. Second, they show the same calculation difficulties whether the problem is presented to them visually or auditorily, and whether they have to respond verbally or in writing, or even merely have to decide whether a proposed operation is true or false. Thus, the calculation deficit is not due to an inability to identify the numbers or to produce the operation result. Third, the deficit often extends to tasks outside of calculation per se, such as comparison or bisection. For instance, patient MAR (Dehaene \& Cohen, 1997) showed a mild impairment in deciding which of two numbers is the larger (16\% errors), and was almost totally unable to decide what number falls in the middle of two others (bisection task: $77 \%$ errors). He easily performed analogous comparison and bisection tasks in non-numerical domains such as days of the week, months, or the alphabet (What is between Tuesday and Thursday? February and April? B and D?). This type of deficits seems best described as a category-specific impairment of the semantic representation and manipulation of numerical quantities (Dehaene \& Cohen, 1997), rather than with the mere clinical label of "acalculia."

In such patients, calculation impairments often co-occur with other deficits, forming a cluster of deficits called Gerstmann's syndrome (Benton, 1992; Gerstmann, 1940), which comprises agraphia, finger agnosia, and left-right distinction difficulties (to which one may often add constructive apraxia). The lesions that cause acalculia of the
Gerstmann's type are typically centred in the depth of the left intraparietal sulcus (Mayer, Martory, Pegna, Landis, Delavelle, \& Annoni, 1999; Takayama, Sugishita, Akiguchi, \& Kimura, 1994). This is compatible with the above brain-imaging results showing intraparietal activation during various numerical manipulation tasks independently of language. Results from a recent brain-imaging study (Simon et al., 2002) shed some light on why the various elements of Gerstmann's syndrome often co-occur following left intraparietal lesions. In this study, fMRI was used to compare, in the same subjects, the localisation of parietal activations during a number subtraction task with those observed during various tasks that also involve the parietal lobe, such as eye or attention movements, finger pointing, hand grasping, and a language task of phoneme detection. The results revealed a systematic topographical organisation of activations and their intersections. In particular, the intraparietal sulcus appears to contains a "four-corners" region in which four areas of activation are juxtaposed: calculation only, calculation and language, manual tasks only, and an area activated during the four visuospatial tasks (eye and attention movements, pointing, and grasping). The simultaneous lesion of those four areas would predictably result in joint impairments of calculation, word processing (possibly including agraphia), finger knowledge and movement, and high-level spatial reference (possibly including understanding of leftright coordinates). Such a joint lesion might be frequent because this cortical territory is jointly irrigated by a branch of the middle cerebral artery, the angular gyrus artery. Inter-individual variability in the boundaries between cortical territories as well as in the branching patterns of this artery would explain that the different elements of Gerstmann's syndrome can be dissociated (Benton, 1961, 1992). Note that this interpretation implies that, contrary to a frequent speculation, Gerstmann's syndrome does not result from a homogeneous impairment to a single representation that would somehow intermingle fingers, numbers, and space (Butterworth, 1999; Gerstmann, 1940; Mayer et al., 1999). Rather, the syndrome may represent a happenstance conjunction of distinct, but dissociable, 
deficits that frequently co-occur due to a common vascularisation, and that are only loosely connected at the functional level due to the overarching spatial and sensorimotor functions of the parietal lobe.

\section{THE LEFT ANGULAR GYRUS AND VERBAL NUMBER MANIPULATIONS}

\section{Neuroimaging evidence}

The left angular gyrus (hereafter AG) is also often activated in neuroimaging studies of number processing (see Figure $1 \mathrm{~b}$ and Table 1 ). This region is left-lateralised and located posterior and inferior to the HIPS (see Figure 2 for their respective locations). A closer look at the types of numerical tasks that activate this region, detailed below, reveals that its functional properties are very different from the properties of the HIPS. The left AG does not seem to be concerned with quantity processing, but shows increasingly greater activation as the task puts greater requirement on verbal processing. We therefore propose that this region is part of the language system, and contributes to number processing only inasmuch as some arithmetic operations, such as multiplication, make particularly strong demands on a verbal coding of numbers.

In support of this hypothesis, the left $A G$ is not merely involved in calculation, but in different types of language-mediated processes such as reading or verbal short-term memory tasks (for reviews, see Fiez \& Petersen, 1998; Paulesu, Frith, \& Frackowiak, 1993; Price, 1998). In Simon et al.'s (2002) fMRI study of six different tasks, the left angular gyrus was the only parietal site where there
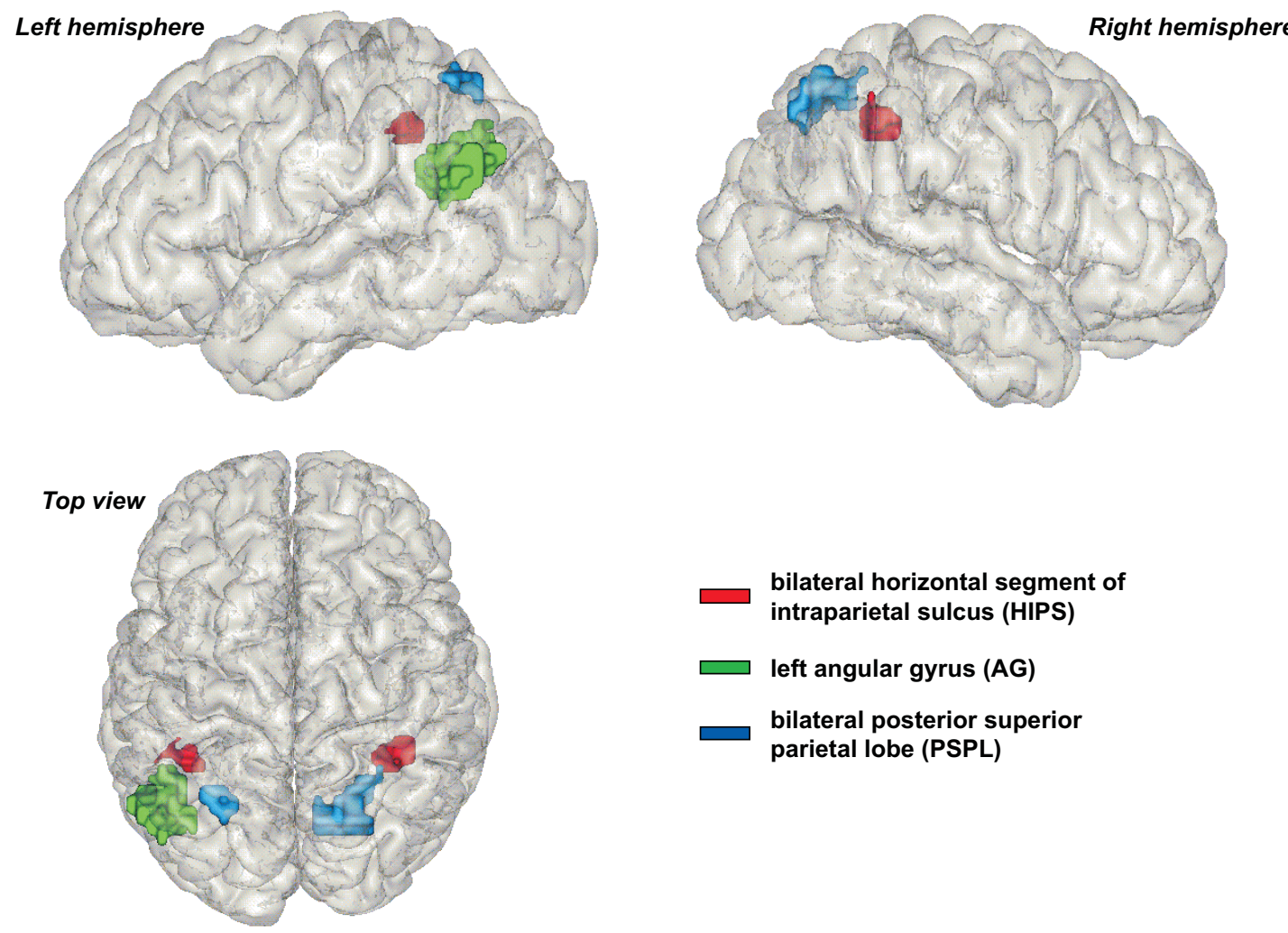

Figure 2. Three-dimensional representation of the parietal regions of interest. For better visualisation, the clusters show all parietal voxels activated in at least $40 \%$ of studies in a given group. 
was overlapping activity for calculation and phoneme detection, but no activation during the other four visuospatial tasks. This clearly indicates that the left $A G$ is not specific for calculation, but jointly recruited by language and calculation processes.

Even within calculation, several studies indicate a modulation of AG activation in direct proportion to the verbal requirements of the task. First, the AG is more active in exact calculation than in approximation (Dehaene et al., 1999). This fits with behavioural data indicating that exact arithmetic facts are stored in a language-specific format in bilinguals, while approximate knowledge is language-independent and shows the classical numerical distance effect associated with the nonverbal quantity system (Xu \& Spelke, 2000). Second, within exact calculation, the left AG shows greater activation for operations that require access to a rote verbal memory of arithmetic facts, such as multiplication, than for operations that are not stored and require some form of quantity manipulation. For instance, the left AG shows increased activation for multiplication relative to both subtraction and number comparison (Chochon et al., 1999; Lee, 2000), for multiplication and division relative to a letter substitution control (Gruber, Indefrey, Steinmetz, \& Kleinschmidt, 2001), and for multidigit mulplication relative to a digit-matching control (Fulbright, Molfese, Stevens, Skudlarski, Lacadie, \& Gore, 2000).

Even within a given operation, such as singledigit addition, the left angular gyrus is more active for small problems with a sum below 10 than for large problems with a sum above 10 (StanescuCosson, Pinel, Van de Moortele, Le Bihan, Cohen, \& Dehaene, 2000). This probably reflects the fact that small addition facts, just like multiplication tables, are stored in rote verbal memory, while behavioural evidence indicates that larger addition problems are often solved by resorting to various semantic elaboration strategies (Dehaene \& Cohen, 1995; Lefevre, 1996).

In summary, the contribution of the left angular gyrus in number processing may be related to the linguistic basis of arithmetical computations. Its contribution seems essential for the retrieval of facts stored in verbal memory, but not for other numerical tasks (like subtraction, number comparison, or complex calculation) that call for a genuinely quantitative representation of numbers and relate more to the intraparietal sulcus.

\section{Neuropsychological evidence: Dissociations between operations}

The finding that the intraparietal sulcus and the angular gyrus exhibit functionally differentiated properties can shed light on the neuropsychology of acalculia. One of the most striking findings is the occurrence of sharp dissociations between arithmetic operations. It is not rare for a patient to be much more severely impaired in multiplication than in subtraction (Cohen \& Dehaene, 2000; Dagenbach \& McCloskey, 1992; Dehaene \& Cohen, 1997; Lampl, Eshel, Gilad, \& Sarova-Pinhas, 1994; Lee, 2000; Pesenti, Seron, \& Van der Linden, 1994; Van Harskamp \& Cipolotti, 2001), while other patients are much more impaired in subtraction than in multiplication (Dehaene \& Cohen, 1997; Delazer \& Benke, 1997; Van Harskamp \& Cipolotti, 2001). Some have proposed that such dissociations reflect random impairments in a system with distinct stores of arithmetic facts for each operation (Dagenbach \& McCloskey, 1992). Here, however, we would like to show that there is much more systematicity behind those observations. Our views suggest that dissociations between operations reflect a single, basic distinction between overlearned arithmetic facts such as the multiplication table, which are stored in rote verbal memory, and the genuine understanding of number meaning that underlies nontable operations such as subtraction (Dehaene \& Cohen, 1997; Delazer \& Benke, 1997; Hittmair-Delazer, Sailer, \& Benke, 1995). According to this interpretation, multiplication requires the integrity of language-based representations of numbers, because multiplication facts are typically learned by rote verbal memorisation. Subtraction, on the other hand, is typically not learned by rote. Although the mechanisms by which simple subtraction problems are resolved are not yet understood, it is likely that some form of internal manipulation of nonverbal quantities on the internal number line is involved, as attested by the fact 
that very simple subtractions are accessible to preverbal infants (Wynn, 1992) and nonhuman primates (Hauser et al., 2000).

Support for this view comes from several lines of research. First, as noted earlier, imaging studies in normals confirm that distinct sites of activations underlie performance in simple multiplication and subtraction (Chochon et al., 1999; Cohen, Dehaene, Chochon, Lehéricy, \& Naccache, 2000; Lee, 2000). Second, all patients in whom subtraction was more impaired than subtraction had left parietal lesions and/or atrophy, most often accompanied by Gerstmann's syndrome, compatible with an impairment to the left HIPS and to the semantic representation of numerical quantities (Dehaene \& Cohen, 1997; Delazer \& Benke, 1997; Van Harskamp \& Cipolotti, 2001). Conversely, although this is not always thoroughly documented, patients in whom multiplication is more impaired than subtraction typically have associated aphasia (e.g., Cohen et al., 2000; Dehaene \& Cohen, 1997). Furthermore, the lesions often spare the intraparietal cortex and can affect multiple regions known to be engaged in language processing, such as the left perisylvian cortices including the inferior parietal lobule (Cohen et al., 2000), the left parieto-temporal carrefour (Lampl et al., 1994), or the left basal ganglia (Dehaene \& Cohen, 1997).

Multiplication impairments with spared subtraction have also been reported in two patients with reading deficits in whom the lesion affected access to the language system from visual symbols (Cohen \& Dehaene, 2000; McNeil \& Warrington, 1994). Amazingly, one of those patients was able to subtract better than she could read the same problems (Cohen \& Dehaene, 2000). This confirms the relative independence of subtraction, but not multiplication, from the language system.

Perhaps the best evidence for a dissociation between quantity processing in the HIPS and verbal number processing in the left AG comes from two studies of the temporary calculation impairments caused by electrical brain stimulation. In one patient with strips of subdural electrodes arranged over the left parietal, superior temporal, and posterior frontal regions, a single electrode site was found whose stimulation systematically disrupted multiplication performance much more than addition performance $(27 \%$ vs. $87 \%$ correct; subtraction was not tested; Whalen, McCloskey, Lesser, \& Gordon, 1997). Although limited information is available on localisation, this electrode was located in the left inferior parietal region, apparently close to the angular gyrus. Interestingly, multiplication performance was worse when the responses were given orally $(27 \%$ correct $)$ than when they were typed with a key pad (64\% correct), suggesting that stimulation also interfered with the verbal coding of numbers.

A second case presented a double dissociation between subtraction and multiplication (Duffau et al., 2002). Cortical stimulation was performed intra-operatively during the resection of a parietooccipital glioma. Two neighbouring sites were found within the left parietal lobe. The first, located within the angular gyrus proper (approximate Talairach coordinates $-50,-60,+30)$, disrupted multiplication but not subtraction when stimulated. The second, located more superiorily and anteriorily within the intraparietal sulcus (TC $-45,-55,+40)$, disrupted subtraction but not multiplication. An intermediate location was also found where stimulation disrupted both operations. The reported coordinates, although imprecise given the distortions possibly induced by the glioma and the surgery, are completely compatible with the dissociated areas of activation observed in functional brain imaging (Chochon et al., 1999; Lee, 2000).

To close on the issue of dissociations between operations, we briefly consider the case of addition (see also Cohen \& Dehaene, 2000). Addition is complex because it can be solved in at least two ways. It is similar to multiplication in that many people have memorised most of the basic addition table (single digit addition facts with a sum below 10). However, addition is also similar to subtraction in that simple addition problems can also be solved by quantity manipulation strategies, something that would be utterly impractical with multiplication. Thus, addition performance is hard to predict. Indeed, in our experience, it varies considerably 
across patients or even within patients, depending on the strategy that they adopt. The only clear prediction from our model is that addition performance cannot dissociate from both subtraction and multiplication together. That is to say, a patient cannot be impaired in addition, but not in subtraction nor in multiplication (since the latter would imply that both the verbal and the quantity circuits are intact); nor can a patient show preserved addition with impaired subtraction and multiplication (since the latter would imply that both systems are impaired).

If dissociations between operations followed a chance pattern, this prediction should be violated in about one third of cases. In fact, however, it is confirmed by essentially all patients to date (10 out of 11 patients: Cohen \& Dehaene, 2000; Dagenbach \& McCloskey, 1992; Dehaene \& Cohen, 1997; Delazer \& Benke, 1997; Lampl et al., 1994; Lee, 2000; Pesenti et al., 1994; Van Harskamp \& Cipolotti, 2001). The only exception (patient FS, Van Harskamp \& Cipolotti, 2001) is worth discussing. Overall, this patient was $96.3 \%$ correct (156/162) in single-digit subtraction and multiplication, but only $61.7 \%$ correct (100/162) in singledigit addition, thus superficially qualifying as a straightforward violation of our hypothesis. However, the pattern of errors in this patient was quite different from other cases of acalculia; $87 \%$ of his addition errors consisted of selecting the wrong operation (he almost always solved the corresponding multiplication problem, e.g., $3+3=9$ ). This is very different from the other two patients reported in the same paper: patient DT, who was impaired in subtraction, made only $12.5 \%$ operation errors, and patient VP, who was impaired in multiplication, only $3.5 \%$.

In a reanalysis, we excluded patient FS's operation errors and analysed only the remaining trials, in which he was presumably really attempting to add the operands. In this way, we can estimate patient FS's conditional success rate in addition, given that he is really trying to add. This success rate is $92.6 \%$ correct (100/108), a value which does not differ from the performance observed in the other two operations $(96.3 \%$ correct). Thus, it can be argued that patient FS experiences little difficulty with arithmetic operations per se, but exhibits a selective deficit in choosing the appropriate operation. Exactly how subjects transform the task instructions and operation signs into the selection of an appropriate information-processing circuit is left largely unspecified in current models. Nevertheless, deficits affecting this tasksetting level should be kept conceptually distinct from the genuine impairments in arithmetical computation itself.

In summary, a review of neuropsychological dissociations between arithmetic operations indicates that it is not necessary to postulate as many brain circuits as there are arithmetical operations (Dagenbach \& McCloskey, 1992). Rather, most if not all cases so far can be accommodated by the postulated dissociation between a quantity circuit (supporting subtraction and other quantitymanipulation operations) and a verbal circuit (supporting multiplication and other rote memorybased operations).

\section{THE POSTERIOR SUPERIOR PARIETAL SYSTEM AND ATTENTIONAL PROCESSES}

\section{Neuroimaging evidence}

A third region, observed bilaterally in the posterior superior parietal lobule (hereafter PSPL), with a frequent mesial extension into the precuneus, is also active in several tasks requiring number manipulations. This region is posterior to the HIPS, and occupies a location superior and mesial to the AG in the superior parietal lobule (see Figure $1 \mathrm{c}$ and Figure 2). It is active during number comparison (Pesenti et al., 2000; Pinel et al., 2001), approximation (Dehaene et al., 1999), subtraction of two digits (Lee, 2000), and counting (Piazza et al., 2002a). It also appears to increase in activation when subjects carry out two operations instead of one (Menon et al., 2000). However, this region is clearly not specific to the number domain. Rather, it also plays a central role in a variety of visuospatial tasks including hand 
reaching, grasping, eye and/or attention orienting, mental rotation, and spatial working memory (Corbetta, Kincade, Ollinger, McAvoy, \& Shulman, 2000; Culham \& Kanwisher, 2001; Simon et al., 2002). For example, Wojciulik and Kanwisher (1999) have observed overlapping activations in this region in three tasks that all shared a component of attention-orienting. Similarly, Simon et al. (2002) observed that this region was activated during eye movement, attention movements, grasping, and pointing.

The contribution of this region to spatial attention and/or eye orienting probably explains its activation during counting, where subjects are sequentially attending to the enumerated objects. However, spatial attention does not seem to explain its activation during purely numerical operations of comparison, approximation, or subtraction. In all of those tasks, number-related activation in the PSPL was observed relative to a control that used the same spatial distribution of stimuli on screen, as well as a very similar motor response.

Obviously, any reconciliation of those sparse and disparate data set must remain tentative. The hypothesis that we would like to propose is that this region, in addition to being involved in attention orienting in space, can also contribute to attentional selection on other mental dimensions that are analogous to space, such as time (Coull \& Nobre, 1998; Wojciulik \& Kanwisher, 1999) or number. Psychological experiments indicate that the core semantic representation of numerical quantity can be likened to an internal "number line," a quasispatial representation on which numbers are organised by their proximity (Dehaene, Bossini, \& Giraux, 1993; Moyer \& Landauer, 1967). It is then conceivable that the same process of covert attention that operates to select locations in space can also be engaged when attending to specific quantities on the number line. Such number-based attention would be particularly needed in tasks that call for the selection of one amongst several quantities, for instance when deciding which of two quantities is the larger (Pesenti et al., 2000; Pinel et al., 2001), or which of two numbers approximately fits an addition problem (Dehaene et al., 1999).

\section{Neuropsychological evidence: Joint impairments of attention and number processing}

Only a few neuropsychological and brain stimulation findings provide some support for our admittedly speculative theory. In a recent study using transcranial magnetic stimulation with normal subjects, Gobel, Walsh, and Rushworth (2001) first located left and right dorsal posterior parietal sites where stimulation interfered with performance in a visual serial search task. The coordinates of those regions correspond to those of the bilateral posterior parietal regions found active in neuroimaging studies of eye and attention orienting (Corbetta et al., 2000; Simon et al., 2002; Wojciulik \& Kanwisher, 1999). They then tested the effect of magnetic stimulation at those locations on a twodigit number comparison task. On stimulated trials, comparison performance was significantly slower. Interestingly, the numerical distance effect itself was still present and relatively unchanged (although stimulation on the left tended to interfere more with numbers close to the reference, particularly those that were larger than the reference). This suggests that the stimulation did not directly interfere with a core representation of numerical quantity, but rather with the response decision process itself. At the very least, this experiment confirms that spatial attention orienting and numerical comparison both engage this parietal region, thus confirming previous brain-imaging evidence (Pinel et al., 2001).

Further support for a close interplay between the representations of space and numbers is provided by a study with unilateral neglect patients (Zorzi, Priftis, \&Umiltà, 2002). It is a well-known, indeed almost a defining feature of those patients that they perform poorly in spatial bisection tests. When asked to locate the middle of a line segment, neglect patients with right parietal lesions tend to indicate a location further to the right, consistent with their failure to attend to the left side of space. Zorzi et al. tested their performance in a numerical bisection task, where they were asked to find the middle of two orally presented numbers. Strikingly, patients erred systematically, often selecting a number far 
larger than the correct answer (e.g., Q: What number falls in between 11 and 19? A: 17). This suggests that spatial attention can be oriented on the left-toright oriented number line, and that this attentionorienting process contributes to the resolution of simple arithmetic problems such as the bisection test. Interestingly, these patients were said not to be acalculic and did not show any deficit in other numerical tasks such as simple arithmetic fact retrieval. Indeed, Vuilleumier and Rafal (1999) demonstrated, on a different group of patients with neglect, that a posterior parietal lesion does not impair the mere quantification of small number of items. Neglect patients were able to estimate numerosity with sets of up to four objects even when some of enumerated items fell in the neglected field. Again, this suggests that attentional and numerical systems are dissociable. However, Zorzi et al.'s finding of “representational neglect" on the numerical continuum indicates that spatial attention processes do contribute to some numerical tasks.

\section{DEVELOPMENTAL DYSCALCULIA AND THE ONTOGENY OF NUMBER REPRESENTATIONS}

Whether or not our functional characterisation of three parietal subsystems is correct, it is an anatomical fact that those activations sites are strikingly reproducible. It is remarkable that the HIPS, AG, and PSPL are systematically activated in different subjects, often from different countries, with different educational strategies and achievements in mathematics (Stevenson \& Stigler, 1992), and with a diversity of linguistic schemes for expressing number (Hurford, 1987). Even the fine dissociation between subtraction and multiplication is reproducible with French vs. Korean subjects (Cohen et al., 2000; Lee, 2000). Such systematicity in the anatomical organisation of parietal numerical processes must be reconciled with the obvious fact that arithmetic is, in part, a recent cultural invention.

Our hypothesis is that the cultural construction of arithmetic is made possible by pre-existing cere- bral circuits that are biologically determined and are adequate to support specific subcomponents of number processing (Dehaene, 1997). This hypothesis supposes an initial prespecialisation of the brain circuits that will ultimately support high-level arithmetic in adults. It implies that it should be possible to identify precursors of those circuits in infancy and childhood. Indeed, quantity processing is present at a very young age. Infants in their first year of life can discriminate collections based on their numerosity (Dehaene et al., 1998a; Starkey \& Cooper, 1980; Wynn, 1992), even when the numbers are as large as 8 vs. 16 (Xu \& Spelke, 2000). Although no brain-imaging evidence is available in infants yet, we speculate that this early numerical ability may be supported by a quantity representation similar to adults' (Dehaene, 1997; Spelke \& Dehaene, 1999). This representation would serve as a foundation for the construction of higher-order arithmetical and mathematical concepts.

The hypothesis of an early emergence of quantity, verbal, and attentional systems leads to several predictions concerning normal and impaired number development:

Brain activation in infancy and childhood. A precursor of the HIPS region should be active in infants and young children during numerosity manipulation tasks. At present, this prediction has only been tested with 5-year-old children in a number comparison task (E. Temple \& Posner, 1998). Eventrelated potentials revealed the scalp signature of a numerical distance effect, with a topography similar to adults, common to numbers presented as Arabic numerals or as sets of dots. There is a clear need to extend those data to an earlier age and with a greater anatomical accuracy.

Developmental dyscalculia and the parietal lobe. Deficits of number processing should be observed in case of early left parietal injury or disorganisation. Developmental dyscalculia is relatively frequent, affecting 3-6\% of children (Badian, 1983; Kosc, 1974; Lewis, Hitch, \& Walker, 1994). We predict that a fraction of those children may suffer from a core conceptual deficit in the numerical domain. Indeed, a "developmental Gerstmann syndrome" 
has been reported (Benson \& Geschwind, 1970; Kinsbourne \& Warrington, 1963; Spellacy \& Peter, 1978; C. M. Temple, 1989, 1991). In those children, dyscalculia is accompanied by most or all of the following symptoms: dysgraphia, left-right disorientation, and finger agnosia, which suggest a neurological involvement of the parietal lobe. Interestingly, even in a sample of 200 normal children, a test of finger knowledge appears to be a better predictor of later arithmetic abilities than is a test of general intelligence (Fayol, Barrouillet, \& Marinthe, 1998).

Two recent reports directly relate developmental dyscalculia to an underlying left parietal disorganisation. Levy, Reis, and Grafman (1999) report the case of an adult with lifelong isolated dyscalculia together with superior intelligence and reading ability, in whom the standard anatomical MRI appeared normal, yet MR spectroscopy techniques revealed a metabolic abnormality in the left inferior parietal area. Similarly, Isaacs, Edmonds, Lucas, and Gadian (2001) used voxel-based morphometry to compare gray matter density in adolescents born at equally severe grades of prematurity, half of whom suffered from dyscalculia. They found a single region of reduced gray matter in the left intraparietal sulcus. The Talairach coordinates of this region $(-39,-39,+45)$ are quite close to the coordinates of the HIPS.

Subtypes of developmental dyscalculia. As in adult acalculia, at least two subtypes of developmental dyscalculia should be observed, and those should be traceable to a differential impairment of quantity vs. language processing circuits. Although several distinctions between subtypes of developmental dyscalculia have been proposed (e.g., Ashcraft, Yamashita, \& Aram, 1992; Geary, Hamson, \& Hoard, 2000; Rourke \& Conway, 1997; C. M. Temple, 1991), most are based on group studies and standardised batteries of tests, which are inappropriate for testing the predicted subtle distinctions between, e.g., subtraction and multiplication. One exception is the single-case study of patient HM (C. M. Temple, 1991), who suffered from developmental phonological dyslexia. His deficit in arithmetic was mostly limited to multiplication facts, while he experienced no difficulty in solving simple addition and subtraction problems with numbers of the same size. Our view predicts that the association of verbal and multiplication impairments observed in this study should be generalisable. Multiplication deficits should be present in cases of dyscalculia accompanied by dysphasia and/or dyslexia, while subtraction and quantity-manipulation deficits should be present in patients with dyscalculia but without any accompanying dyslexia or language retardation. Although this proposal remains largely untested, Geary et al. (2000) do report interesting differences between developmental dyscalculics with or without associated dyslexia. When faced with the same simple addition problems, nondyslexics tend to use fact retrieval much more often than do dyslexics, who rather use finger-counting strategies. This is consistent with the hypothesis that an impairment of rote verbal memory is partially responsible for dyscalculia in children with dyslexia.

Genetics of developmental dyscalculia. If the biological predisposition view is correct, specific combinations of genes should be involved in setting up the internal organisation of the parietal lobe and, in particular, the distinction between quantity and language circuits. Thus, it should be possible to identify dyscalculias of genetic origin. The available data, indeed, indicate that when a child is dyscalculic, other family members are also frequently affected, suggesting that genetic factors may contribute to the disorder (Shalev et al., 2001). Although the search for dyscalculias of genetic origin has only very recently begun, the possibility that Turner syndrome may conform to this typology has recently attracted attention. Turner syndrome is a genetic disorder characterised by partial or complete absence of one $\mathrm{X}$ chromosome in a female individual. The disorder occurs in approximately 1 girl in 2000 and is associated with well-documented physical disorders and abnormal oestrogen production and pubertal development. The cognitive profile includes deficits in visual memory, visual-spatial and attentional tasks, and social relations, in the context of a normal verbal IQ (Rovet, 1993). Most interestingly in the present context is 
the documentation of a mild to severe deficit in mathematics, particularly clear in arithmetic (Mazzocco, 1998; Rovet, Szekely, \& Hockenberry, 1994; C. M. Temple \& Marriott, 1998).

Anatomically, the data suggest possible bilateral parieto-occipital dysfunction in Turner syndrome. A positron emission tomography study of five adult women demonstrated a glucose hypometabolism in bilateral parietal and occipital regions (Clark, Klonoff, \& Hadyen, 1990). Two anatomical MR studies, one with 18 and the other with 30 affected women, demonstrated bilateral reductions in parieto-occipital brain volume, together with other subcortical regions (Murphy et al., 1993; see also Reiss et al., 1993; Reiss, Mazzocco, Greenlaw, Freund, \& Ross, 1995). Interestingly, the phenotype of Turner syndrome can differ depending on whether the remaining $\mathrm{X}$ chromosome is of paternal or maternal origin (Xm or Xp subtypes; Bishop, Canning, Elgar, Morris, Jacobs, \& Skuse, 2000; Skuse, 2000; Skuse et al., 1997). Such a genomic imprinting effect was first demonstrated on tests of social competence (Skuse et al., 1997). It will be interesting to see if a similar effect exists in the arithmetic domain.

\section{CONCLUSION}

We have reviewed the evidence for a subdivision of calculation-related processes in the parietal lobe. A broader discussion of the specificity of the number processing system should also consider the satellite systems that serve as input and outputs to calculation processes. At the visual identification level, pure alexic patients who fail to read words often show a largely preserved ability to read and process digits (Cohen \& Dehaene, 1995; Déjerine, 1891, 1892). Conversely, a case of impaired number reading with preserved word reading is on record (Cipolotti, Warrington, \& Butterworth, 1995). In the writing domain, severe agraphia and alexia may be accompanied by a fully preserved ability to write and read Arabic numbers (Anderson, Damasio, \& Damasio, 1990). Even within the speech production system, patients who suffer from random phoneme substitutions, thus resulting in the pro- duction of an incomprehensible jargon, may produce jargon-free number words (Cohen, Verstichel, \&Dehaene, 1997). These dissociations, however, need not imply a distinct semantic system for number. Rather, they can probably be explained by considering that the particular syntax of number words and the peculiarities of the positional notation for Arabic numeral place special demands on visual recognition, speech production, and writing systems.

Even within the parietal lobe, our review of number-related activations suggests that much of the human capacity for number processing relies on representations and processes that are not specific to the number domain. At least two of the parietal circuits that we have described, the posterior superior parietal attention system and the left angular verbal system, are thought to be associated with broader functions than mere calculation. The third circuit, in the bilateral horizontal intraparietal region (HIPS), is a more plausible candidate for domain specificity. As reviewed above, it is systematically activated during mental arithmetic; it is more activated by number words than by other words such as names of animals; and its activation increases with the amount or duration of quantity manipulation required, but is completely independent of the notation used for numbers. Still, we are reluctant to use the term "category-specific" for this brain region, and prefer the terms "core quantity system" or "number-essential" region instead. For a purely empirical point of view, deciding whether a given region is "specific" for numbers seems an extremely difficulty enterprise. Testing for specificity would seem to require a systematic comparison of the target category (e.g., number) against a potentially infinite list of alternatives. It is also complicated by the limited resolution of brainimaging techniques, which cannot yet resolve the fine-grained neuronal and columnar organisation of human cortex. Comparison of group studies, as was done here, may overestimate the amount of overlap between tasks. Studies of multiple tasks within the same subjects will be required to examine whether (1) the very same voxels can be activated by multiple quantity-related paradigms, and (2) those voxels cannot be activated by any other non- 
numerical operation. Because such studies are lacking (although see Simon et al., 2002), it is still premature to conclude for or against categoryspecificity in number semantics.

\section{REFERENCES}

Anderson, S. W., Damasio, A. R., \& Damasio, H. (1990). Troubled letters but not numbers. Domain specific cognitive impairments following focal damage in frontal cortex. Brain, 113, 749-766.

Ashcraft, M. H., Yamashita, T. S., \& Aram, D. M. (1992). Mathematics performance in left and right brain-lesioned children and adolescents. Brain and Cognition, 19, 208-252.

Badian, N. A. (1983). Dyscalculia and nonverbal disorders of learning. In H. R. Myklebust (Ed.), Progress in learning disabilities (Vol. 5, pp. 235-264). New York: Stratton.

Benson, D. F., \& Geschwind, N. (1970). Developmental Gerstmann syndrome. Neurology, 20, 293-298.

Benton, A. L. (1961). The fiction of the Gerstmann syndrome. Journal of Neurology, 24, 176-181.

Benton, A. L. (1992). Gerstmann's syndrome. Archives of Neurology, 49, 445-447.

Bishop, D. V., Canning, E., Elgar, K., Morris, E., Jacobs, P. A., \& Skuse, D. H. (2000). Distinctive patterns of memory function in subgroups of females with Turner syndrome: Evidence for imprinted loci on the X-chromosome affecting neurodevelopment. Neuropsychologia, 38, 712-721.

Burbaud, P., Camus, O., Guehl, D., Bioulac, B., Caille, J. M., \& Allard, M. (1999). A functional magnetic resonance imaging study of mental subtraction in human subjects. Neuroscience Letters, 273, 195-199.

Butterworth, B. (1999). The mathematical brain. London: Macmillan.

Butterworth, B., Cappelletti, M., \& Kopelman, M. (2001). Category specificity in reading and writing: The case of number words. Nature Neuroscience, 4, 784-786.

Cappelletti, M., Butterworth, B., \& Kopelman, M. (2001). Spared numerical abilities in a case of semantic dementia. Neuropsychologia, 39, 1224-39.

Chochon, F., Cohen, L., van de Moortele, P. F., \& Dehaene, S. (1999). Differential contributions of the left and right inferior parietal lobules to number processing. Journal of Cognitive Neuroscience, 11, 617630.
Cipolotti, L., Butterworth, B., \& Denes, G. (1991). A specific deficit for numbers in a case of dense acalculia. Brain, 114, 2619-2637.

Cipolotti, L., Warrington, E. K., \& Butterworth, B. (1995). Selective impairment in manipulating arabic numerals. Cortex, 31, 73-86.

Clark, C., Klonoff, H., \& Hadyen, M. (1990). Regional cerebral glucose metabolism in Turner syndrome. Canadian Journal of Neurological Sciences, 17, 140144.

Cohen, L., \&Dehaene, S. (1995). Number processing in pure alexia: The effect of hemispheric asymmetries and task demands. NeuroCase, 1, 121-137.

Cohen, L., \& Dehaene, S. (1996). Cerebral networks for number processing: Evidence from a case of posterior callosal lesion. NeuroCase, 2, 155-174.

Cohen, L., \& Dehaene, S. (2000). Calculating without reading: Unsuspected residual abilities in pure alexia. Cognitive Neuropsychology, 17, 563-583.

Cohen, L., Dehaene, S., Chochon, F., Lehéricy, S., \& Naccache, L. (2000). Language and calculation within the parietal lobe: A combined cognitive, anatomical and fMRI study. Neuropsychologia, 38, 1426-1440.

Cohen, L., Verstichel, P., \& Dehaene, S. (1997). Neologistic jargon sparing numbers: a category specific phonological impairment. Cognitive Neuropsychology, 14, 1029-1061.

Corbetta, M., Kincade, J. M., Ollinger, J. M., McAvoy, M. P., \& Shulman, G. L. (2000). Voluntary orienting is dissociated from target detection in human posterior parietal cortex. Nature Neuroscience, 3, 292-297.

Coull, J. T., \& Nobre, A. C. (1998). Where and when to pay attention: The neural systems for directing attention to spatial locations and to time intervals as revealed by both PET and fMRI. Journal of Neuroscience, 18, 7426-7435.

Culham, J. C., \& Kanwisher, N. G. (2001). Neuroimaging of cognitive functions in human parietal cortex. Current Opinions in Neurobiology, 11, 157-63.

Dagenbach, D., \& McCloskey, M. (1992). The organization of arithmetic facts in memory: Evidence from a brain-damaged patient. Brain and Cognition, 20, 345-366.

Dehaene, S. (1992). Varieties of numerical abilities. Cognition, 44, 1-42.

Dehaene, S. (1995). Electrophysiological evidence for category-specific word processing in the normal human brain. NeuroReport, 6, 2153-2157.

Dehaene, S. (1996). The organization of brain activations in number comparison: Event-related potentials 
and the additive-factors methods. Journal of Cognitive Neuroscience, 8, 47-68.

Dehaene, S. (1997). The number sense. New York: Oxford University Press.

Dehaene, S., Bossini, S., \& Giraux, P. (1993). The mental representation of parity and numerical magnitude. Journal of Experimental Psychology: General, 122, 371-396.

Dehaene, S., \& Cohen, L. (1995). Towards an anatomical and functional model of number processing. Mathematical Cognition, 1, 83-120.

Dehaene, S., \& Cohen, L. (1997). Cerebral pathways for calculation: Double dissociation between rote verbal and quantitative knowledge of arithmetic. Cortex, 33, 219-250.

Dehaene, S., Dehaene-Lambertz, G., \& Cohen, L. (1998a). Abstract representations of numbers in the animal and human brain. Trends in Neuroscience, 21, 355-361.

Dehaene, S., \& Marques, F. (2002). Cognitive euroscience: Scalar variability in price estimation and the cognitive consequences of switching to the euro. Quarterly Journal of Experimental Psychology, 55A, 705-731.

Dehaene, S., Naccache, L., Le Clec'H, G., Koechlin, E., Mueller, M., Dehaene-Lambertz, G., Van de Moortele, P. F., \& Le Bihan, D. (1998b). Imaging unconscious semantic priming. Nature, 395, 597600.

Dehaene, S., Spelke, E., Stanescu, R., Pinel, P., \& Tsivkin, S. (1999). Sources of mathematical thinking: Behavioral and brain-imaging evidence. Science, 284, 970-974.

Dehaene, S., Tzourio, N., Frak, V., Raynaud, L., Cohen, L., Mehler, J., \& Mazoyer, B. (1996). Cerebral activations during number multiplication and comparison: A PET study. Neuropsychologia, 34, 1097-1106.

Déjerine, J. (1891). Sur un cas de cécité verbale avec agraphie suivi d'autopsie. Mémoires de la Société de Biologie, 3, 197-201.

Déjerine, J. (1892). Contribution à l'étude anatomopathologique et clinique des différentes variétés de cécité verbale. Mémoires de la Société de Biologie, 4, 6190.

Delazer, M., \& Benke, T. (1997). Arithmetic facts without meaning. Cortex, 33, 697-710.

Duffau, H., Denvil, D., Lopes, M., Gasparini, F., Cohen, L., Capelle, L., et al. (2002). Intraoperative mapping of the cortical areas involved in multiplication and subtraction: An electrostimulation study in a patient with a left parietal glioma. Journal of Neurology, Neurosurgery, and Psychiatry, 73, 733-738.

Fayol, M., Barrouillet, P., \& Marinthe, X. (1998). Predicting arithmetical achievement from neuropsychological performance: A longitudinal study. Cognition, 68, B63-B70.

Fiez, J. A., \& Petersen, S. E. (1998). Neuroimaging studies of word reading. Proceedings of the National Academy of Sciences USA, 95, 914-921.

Fulbright, R. K., Molfese, D. L., Stevens, A. A., Skudlarski, P., Lacadie, C. M., \& Gore, J. C. (2000). Cerebral activation during multiplication: A functional MR imaging study of number processing. American Journal of Neuroradiology, 21, 1048-1054.

Geary, D. C., Hamson, C. O., \& Hoard, M. K. (2000). Numerical and arithmetical cognition: A longitudinal study of process and concept deficits in children with learning disability. Journal of Experimental Child Psychology, 77, 236-263.

Gerstmann, J. (1940). Syndrome of finger agnosia, disorientation for right and left, agraphia, and acalculia. Archives of Neurology and Psychiatry, 44, 398-408.

Gobel, S., Walsh, V., \& Rushworth, M. F. (2001). The mental number line and the human angular gyrus. Neuroimage, 14, 1278-1289.

Gruber, O., Indefrey, P., Steinmetz, H., \& Kleinschmidt, A. (2001). Dissociating neural correlates of cognitive components in mental calculation. Cerebral Cortex, 11, 350-359.

Hauser, M. D., Carey, S., \&Hauser, L. B. (2000). Spontaneous number representation in semi-free-ranging rhesus monkeys. Proceedings of the Royal Society of London B, Biological Science, 267, 829-833.

Hécaen, H., Angelergues, R., \& Houillier, S. (1961). Les variétés cliniques des acalculies au cours des lésions rétro-rolandiques: Approche statistique du problème. Revue Neurologique, 105, 85-103.

Henschen, S. E. (1919). Über Sprach- Musik- und Rechenmechanismen und ihre Lokalisationen im Grosshirn. Zeitschrift für die desamte Neurologie und Psychiatrie, 52, 273-298.

Hittmair-Delazer, M., Sailer, U., \& Benke, T. (1995). Impaired arithmetic facts but intact conceptual knowledge: A single case study of dyscalculia. Cortex, 31, 139-147.

Hurford, J. R. (1987). Language and number. Oxford: Basil Blackwell.

Isaacs, E. B., Edmonds, C. J., Lucas, A., \& Gadian, D. G. (2001). Calculation difficulties in children of very 


\section{DEHAENE ET AL.}

low birthweight: A neural correlate. Brain, 124, 1701-1707.

Kiefer, M., \& Dehaene, S. (1997). The time course of parietal activation in single-digit multiplication: Evidence from event-related potentials. Mathematical Cognition, 3, 1-30.

Kinsbourne, M., \& Warrington, E. K. (1963). The developmental Gerstmann syndrome. Archives of Neurology, 8, 490.

Kosc, L. (1974). Developmental dyscalculia. Journal of Learning Disabilities, 7, 165-177.

Lampl, Y., Eshel, Y., Gilad, R., \& Sarova-Pinhas, I. (1994). Selective acalculia with sparing of the subtraction process in a patient with left parietotemporal hemorrhage. Neurology, 44, 1759-1761.

Langdon, D. W., \& Warrington, E. K. (1997). The abstraction of numerical relations: A role for the right hemisphere in arithmetic? Journal of International Neuropsychological Society, 3, 260-268.

Le Clec'H, G., Dehaene, S., Cohen, L., Mehler, J., Dupoux, E., Poline, J. B., Lehericy, S., Van de Moortele, P. F., \& Le Bihan, D. (2000). Distinct cortical areas for names of numbers and body parts independent of language and input modality. Neuroimage, 12, 381-391.

Lee, K. M. (2000). Cortical areas differentially involved in multiplication and subtraction: A functional magnetic resonance imaging study and correlation with a case of selective acalculia. Annals of Neurology, 48, 657-661.

Lee, K. M., \& Kang, S. Y. (2002). Arithmetic operation and working memory: Differential suppression in dual tasks. Cognition, 83, B63-B68.

Lefevre, J.-A. (1996). Selection of procedures in mental addition: Reassessing the problem-size effect in adults. Journal of Experimental Psychology: Learning, Memory, and Cognition, 22, 216-230.

Levy, L. M., Reis, I. L., \& Grafman, J. (1999). Metabolic abnormalities detected by H-MRS in dyscalculia and dysgraphia. Neurology, 53, 639-641.

Lewis, C., Hitch, G. J., \&Walker, P. (1994). The prevalence of specific arithmetic difficulties and specific reading difficulties in 9- and 10-year-old boys and girls. Journal of Child Psychology and Psychiatry, 35, 283-292.

Mayer, E., Martory, M. D., Pegna, A. J., Landis, T., Delavelle, J., \& Annoni, J. M. (1999). A pure case of Gerstmann syndrome with a subangular lesion. Brain, 122, 1107-1120.
Mazzocco, M. M. (1998). A process approach to describing mathematics difficulties in girls with Turner syndrome. Pediatrics, 102, 492-496.

McNeil, J. E., \& Warrington, E. K. (1994). A dissociation between addition and subtraction with written calculation. Neuropsychologia, 32, 717-728.

Menon, V., Rivera, S. M., White, C. D., Glover, G. H., \& Reiss, A. L. (2000). Dissociating prefrontal and parietal cortex activation during arithmetic processing. NeuroImage, 12, 357-365.

Moyer, R. S., \& Landauer, T. K. (1967). Time required for judgements of numerical inequality. Nature, 215, 1519-1520.

Murphy, D. G., DeCarli, C., Daly, E., Haxby, J. V., Allen, G., White, B. J., McIntosh, A. R., Powell, C. M., Horwitz, B., Rapoport, S. I., et al. (1993). Xchromosome effects on female brain: A magnetic resonance imaging study of Turner's syndrome. Lancet, 342, 1197-1200.

Naccache, L., \& Dehaene, S. (2001). The priming method: Imaging unconscious repetition priming reveals an abstract representation of number in the parietal lobes. Cerebral Cortex, 11, 966-974.

Paulesu, E., Frith, C. D., \& Frackowiak, R. S. J. (1993). The neural correlates of the verbal component of working memory. Nature, 362, 342-345.

Pesenti, M., Seron, X., \& Van der Linden, M. (1994). Selective impairment as evidence for mental organisation of arithmetical facts: $\mathrm{BB}$, a case of preserved subtraction? Cortex, 30, 661-671.

Pesenti, M., Thioux, M., Seron, X., \& De Volder, A. (2000). Neuroanatomical substrates of arabic number processing, numerical comparison, and simple addition: A PET study. Journal of Cognitive Neuroscience, 12, 461-479.

Piazza, M., Mechelli, A., Butterworth, B., \& Price, C. J. (2002a). Are subitizing and counting implemented as separate or functionally overlapping processes? Neuroimage, 15, 435-446.

Piazza, M., Mechelli, A., Price, C., \& Butterworth, B. (2002b). The quantifying brain: Functional neuroanatomy of numerosity estimation and counting. Manuscript submitted for publication.

Pinel, P., Dehaene, S., Riviere, D., \& LeBihan, D. (2001). Modulation of parietal activation by semantic distance in a number comparison task. Neuroimage, 14, 1013-1026.

Price, C. (1998). The functional anatomy of word comprehension and production. Trends in Cognitive Science, 2, 281-288. 
Reiss, A. L., Freund, L., Plotnick, L., Baumgardner, T., Green, K., Sozer, A. C., Reader, M., Boehm, C., \& Denckla, M. B. (1993). The effects of X monosomy on brain development: Monozygotic twins discordant for Turner's syndrome. Annals of Neurology, 34, 95107.

Reiss, A. L., Mazzocco, M. M., Greenlaw, R., Freund, L. S., \& Ross, J. L. (1995). Neurodevelopmental effects of X monosomy: A volumetric imaging study. Annals of Neurology, 38, 731-738.

Roland, P. E., \& Friberg, L. (1985). Localization of cortical areas activated by thinking. Journal of Neurophysiology, 53, 1219-1243.

Rosselli, M., \& Ardila, A. (1989). Calculation deficits in patients with right and left hemisphere damage. Neuropsychologia, 27, 607-617.

Rourke, B. P., \& Conway, J. A. (1997). Disabilities of arithmetic and mathematical reasoning. Perspectives from neurology and neuropsychology. Journal of Learning Disabilities, 30, 34-46.

Rovet, J. F. (1993). The psychoeducational characteristics of children with Turner syndrome. Journal of Learning Disabilities, 26, 333-341.

Rovet, J., Szekely, C., \& Hockenberry, M. N. (1994). Specific arithmetic calculation deficits in children with Turner syndrome. Journal of Clinical Experimental Neuropsychology, 16, 820-839.

Rueckert, L., Lange, N., Partiot, A., Appollonio, I., Litvar, I., Le Bihan, D., \& Grafman, J. (1996). Visualizing cortical activation during mental calculation with functional MRI. NeuroImage, 3, 97-103.

Seymour, S. E., Reuter-Lorenz, P. A., \& Gazzaniga, M. S. (1994). The disconnection syndrome: Basic findings reaffirmed. Brain, 117, 105-115.

Shalev, R. S., Manor, O., Kerem, B., Ayali, M., Badichi, N., Friedlander, Y., \& Gross-Tsur, V. (2001). Developmental dyscalculia is a familial learning disability. Journal of Learning Disabilities, 34, 59-65.

Simon, O., Cohen, L., Mangin, J. F., Bihan, D. L., \& Dehaene, S. (2002). Topographical layout of hand, eye, calculation and language related areas in the human parietal lobe. Neuron, 33, 475-487.

Skuse, D. H. (2000). Imprinting, the X-chromosome, and the male brain: Explaining sex differences in the liability to autism. Pediatric Research, 47, 9-16.

Skuse, D. H., James, R. S., Bishop, D. V., Coppin, B., Dalton, P., Aamodt-Leeper, G., Bacarese-Hamilton, M., Creswell, C., McGurk, R., \& Jacobs, P. A. (1997). Evidence from Turner's syndrome of an imprinted X-linked locus affecting cognitive function. Nature, 387, 705-708.

Spelke, E., \& Dehaene, S. (1999). On the foundations of numerical thinking: Reply to Simon. Trends in Cognitive Science, 3, 365-366.

Spelke, E. S., \& Tsivkin, S. (2001). Language and number: A bilingual training study. Cognition, 78, 45-88.

Spellacy, F., \& Peter, B. (1978). Dyscalculia and elements of the developmental Gerstmann syndrome in school children. Cortex, 14, 197-206.

Stanescu-Cosson, R., Pinel, P., Van de Moortele, P.-F., Le Bihan, D., Cohen, L., \& Dehaene, S. (2000). Cerebral bases of calculation processes: Impact of number size on the cerebral circuits for exact and approximate calculation. Brain, 123, 2240-2255.

Starkey, P., \& Cooper, R. G. (1980). Perception of numbers by human infants. Science, 210, 1033-1035.

Stevenson, H. W., \& Stigler, J. W. (1992). The learning gap. New York: Simon \& Schuster.

Takayama, Y., Sugishita, M., Akiguchi, I., \& Kimura, J. (1994). Isolated acalculia due to left parietal lesion. Archives of Neurology, 51, 286-291.

Temple, C. M. (1989). Digit dyslexia: A categoryspecific disorder in development dyscalculia. Cognitive Neuropsychology, 6, 93-116.

Temple, C. M. (1991). Procedural dyscalculia and number fact dyscalculia: Double dissociation in developmental dyscalculia. Cognitive Neuropsychology, 8, 155-176.

Temple, C. M., \& Marriott, A. J. (1998). Arithmetic ability and disability in Turner's syndrome: A cognitive neuropsychological analysis. Developmental Neuropsychology, 14, 47-67.

Temple, E., \& Posner, M. I. (1998). Brain mechanisms of quantity are similar in 5-year-olds and adults. Proceedings of the National Academy of Science USA, 95, 7836-7841.

Thioux, M., Pesenti, M., De Volder, A., \& Seron, X. (2002). Category-specific representation and processing of numbers and animal names across semantic tasks: A PET study. NeuroImage, 13, (6 suppl. 2/2), S617.

Thioux, M., Pillon, A., Samson, D., De Partz, M.-P., Noel, M.-P., \& Seron, X. (1998). The isolation of numerals at the semantic level. NeuroCase, 4, 371389.

Van Harskamp, N. J., \& Cipolotti, L. (2001). Selective impairments for addition, subtraction and multiplica- 
tion. Implications for the organisation of arithmetical facts. Cortex, 37, 363-88.

Vuilleumier, P., \& Rafal, R. (1999). "Both" means more than "two": Localizing and counting in patients with visuospatial neglect. Nature Neuroscience, 2, 783-784.

Whalen, J., McCloskey, M., Lesser, R. P., \& Gordon, B. (1997). Localizing arithmetic processes in the brain: Evidence from transient deficit during cortical stimulation. Journal of Cognitive Neuroscience, 9, 409-417.

Wojciulik, E., \& Kanwisher, N. (1999). The generality of parietal involvement in visual attention. Neuron, 23, 747-764.
Wynn, K. (1992). Addition and subtraction by human infants. Nature, 358, 749-750.

Xu, F., \& Spelke, E. S. (2000). Large number discrimination in 6-month-old infants. Cognition, 74, B1B11.

Zago, L., Pesenti, M., Mellet, E., Crivello, F., Mazoyer, B., \& Tzourio-Mazoyer, N. (2001). Neural correlates of simple and complex mental calculation. NeuroImage, 13, 314-327.

Zorzi, M., Priftis, K., \& Umiltà, C. (2002). Brain damage: Neglect disrupts the mental number line. Nature, 417(6885), 138-139. 